\title{
Mathematical Modelling on Lamprey GnRH-III using Autoregressive Logistic Processes
}

\author{
S. Lakshmi \\ Department of Mathematics, K.N. Govt. Arts \\ College for Women, Thanjavur, Tamilnadu, India
}

\author{
R. Karthik \\ Department of Mathematics, Velammal Engineering \\ College, Chennai, Tamilnadu, India
}

\begin{abstract}
In this paper a mathematical model is developed based on current biological concepts of the lamprey GnRH-III involved in LH and FSH concentration. The physiologic role played by GnRH-III on gonadotropin secretion in mammalian species is controversial. Although GnRH-III is a weak GnRH agonist, early research in mammalian species suggested that GnRH-III can selectively stimulate the secretion of FSH without changing concentration of LH. Statistical analysis was carried out using power logistics, the diagnostic values of FSH , LH as well as LH/FSH ratio at different time points during $\mathrm{GnRH}$ test were evaluated using logistic processes.
\end{abstract}

Keywords: Autoregressive Logistic processes, Luteinizing hormone, Follicle-stimulating hormone, Simulation, Stochastic model.

\section{AMS Classification 62N05,62M10,65C20。}

\section{INTRODUCTION}

Among these isoforms, GnRH-III has a substantial antiproliferative effect on several cancer cell lines. The physiologic role played by GnRH-III on gonadotropin secretion in mammalian species is controversial [1 ]. Although GnRH-III is a weak GnRH agonist, early research in mammalian species suggested that $1 \mathrm{GnRH}-\mathrm{III}$ can selectively stimulate the secretion of FSH without changing concentration of LH. In rodents, IGnRH-III significantly increased FSH concentrations in a dose-dependent manner when using anterior pituitaries at $\left(10^{-9}\right.$ to $\left.10^{-4}\right) \mathrm{M}$ concentrations. In contrast, LH concentrations were affected only when the highest doses of 1GnRH-III $\left(10^{-6}\right.$ to $\left.10^{-4}\right) \mathrm{M}$ were used [6]. Intravenous infusion of 1GnRH-III also increased FSH without changes in

LH concentrations [10]. Subsequently, data from the same

laboratory reported the isolation of a FSH-releasing factor (RF) obtained from the stalk-median eminence of rats. The FSHRF was associated with lGnRH-III, and had the ability to interact with a putative receptor to selectively release FSH . These data and that from other non-traditional sources

[7] suggest that 1GnRH-III is a potent and specific FSHreleasing peptide. However, other lines of research have raised questions about the ability of GnRH-III to selectively secrete FSH in rodents. The presence of $1 \mathrm{GnRH}-\mathrm{III}$ in the brain of rats was identified by immunocytochemistry and subsequently localized in the dorsomedial preoptic area of the brain and colocalized with mGnRH [1]. However, IGnRH-III was not detected in rats and other rodents by reverse-phaseHPLC followed by RIA, or by performing two successive HPLC steps to prevent the coelution of GnRH peptides
.Similarly, when rat pituitary cells were perfused with $1 \mathrm{GnRH}-$ III or mGnRH $\left(10^{-9}\right.$ to $\left.10^{-6}\right) \mathrm{M}, 1 \mathrm{GnRH}$-III was 1,000 fold less active in releasing $\mathrm{LH}$ than $\mathrm{mGnRH}$. Moreover, when rat pituitary cells were perfused with doses $\left(10^{-7}\right.$ to $\left.10^{-5}\right) \mathrm{M}$ of $1 \mathrm{GnRH}-\mathrm{III}$, gonadotropin secretion was increased without any indication of a selective secretion of FSH (Kovacs et al., 2002). These data is in agreement with in vitro results obtained from rat hemipituitaries incubated with doses $\left(10^{-9}\right.$ to $\left.10^{-7}\right) \mathrm{M}$ of $1 \mathrm{GnRH}-\mathrm{III}$. The contradictory results obtained by different laboratories, may be explained by experimental condition, the In thinfluence of the presence or absence of steroid in the in vivo models, and data interpretation [1].

In addition to the information provided above, other areas of investigation have stressed the need to reconsider the traditional conjecture that a single GnRH molecule controls reproduction .Briefly, lesions to the median eminence (ME) of castrated male rats suppressed LH but not FSH pulses, while animals with posterior to mid-ME lesions had no FSH pulses but maintained LH episodic releases . Similarly, ablation of the dorsal anterior hypothalamus of ovariectomized rats suppressed FSH pulses but not LH [6]. These results raise the possibility that another form of GnRH may contribute nontraditionally to the control of reproductive function or may take part in an important neuroendocrine role. The nature of episodic FSH secretion in portal blood cannot be accounted completely by changes in GnRH secretion. When infused with doses $(0.055$, to $1.1 \mathrm{mg} / \mathrm{kg} \mathrm{BW})$ of $1 \mathrm{GnRH}-\mathrm{III}$. Higher doses $(4.4 \mathrm{mg} / \mathrm{kg} \mathrm{BW})$ released LH but not FSH. Similarly, in vitro doses $\left(10^{-7}\right.$ to $\left.10^{-6}\right) \mathrm{M}$ of $1 \mathrm{GnRH}$-III elicited a nonselective increase of LH and FSH, while lower doses $\left(10^{-9}\right.$ to $\left.10^{-8}\right) \mathrm{M}$ were not associated with gonadotropin secretion in bovine adenohypophyseal cells. A clear and unbiased interpretation of the discordant results observed in cattle is difficult. Reagents (RIAs) used in both laboratories to evaluate LH and FSH were provided by the National Hormone and Pituitary Program.Thus, it is unlikely that differences can be attributed to the ability of a particular RIA to detect FSH concentrations [10]. It is concluded that lGnRH-III is a weak GnRH agonist, and at high doses, IGnRH-III has the ability to release LH but not FSH in barrows. Similar findings were also obtained in gilts that were infused(i.m.) with a synthetic lGnRH-III product . In Lamprey, 1GnRH-III significantly increased FSH concentrations in a dose-dependent manner when using anterior pituitaries at $\left(10^{-1}\right.$ to $\left.10^{-10}\right) \mathrm{M}$ concentrations. $[1,7]$ 


\section{MATHEMATICAL MODELS}

A random variable $\mathrm{X}$ has a logistic distribution with location parameter $\alpha \in(0,1]$ and scale parameter $\sigma(>0)$ if

$f_{X}(x)=\left\{1+\exp \left[-\left(\frac{x-\alpha}{\sigma}\right)\right]\right\}^{-1}-\infty<x<\infty$

In such a case $X_{n} \square \mathrm{F}(0, \alpha)$ is written. In particular the conditional mean and variance of such a random variable are given by

$$
E(X)=\alpha^{j} x
$$

and

$\operatorname{vax}(x)=\frac{\left(1-\alpha^{2 j}\right) \pi^{2}}{3}$

The basic logistic process introduced in [9] is defined in the following manner. Let $\left\{\xi_{n}\right\}_{n=0}^{\infty}$ be a sequence of independent identically distributed ( i.i.d.) random variables with common distribution(2.1). Define a Markov process $\left\{X_{n}\right\}_{n=0}^{\infty}$ by

$X_{n+1}=X_{n}-\log \beta$ w.p. $\beta$

$=\min \left\{X_{n}-\log \beta, \xi_{n+1}\right\} w \cdot p 1-\beta$

And Here $\beta$ is a parameter with range $[0,1)$. Straightforward conditioning leads to the conclusion that $X_{n} \approx \mathrm{F}(0, \sigma), \forall n$, i.e. a completely stationary Markov process with logistic marginals. It is convenient to define

$\mathbf{Z}_{n-1}=\frac{X_{n-1}-\mu}{\sigma}, \quad n=1,2,3 \ldots$

The process $\{\square\}_{n=0}^{\infty}$ may be called a standard logistic process. Distributional properties of the process $\left\{X_{n}\right\}$ are readily deduced from those of the standard process $\left\{\mathbf{Z}_{n}\right\}$ for which we have a standard logistic marginal distribution, i.e. $\mathbf{Z}_{n} \approx \mathrm{F}(0, \sigma) \mathrm{n}=0,1,2, \ldots$ By conditioning on $\mathbf{Z}_{n}$ and using the joint survival function of $\left(\mathbf{Z}_{n} \mathbf{Z}_{n+1}\right)$ is readily obtained. Thus

$$
P\left(\mathbf{Z}_{n}>z_{n}, \mathbf{Z}_{n+1}>z_{n+1}\right)=\frac{\left(1+\beta \exp \left(z_{n+1}\right)\right)}{\left(1+\beta \exp \left(z_{n+1}\right)\right)\left(1+\max \left[\exp \left(z_{n+1}\right), \beta \exp \left(z_{n+1}\right)\right]\right)}
$$

The correlation between $\mathbf{Z}_{n}$ and $\mathbf{Z}_{n+1}$ may be evaluated as follows:

$$
\begin{aligned}
& E\left(\mathbf{Z}_{n-1}\left(\mathbf{Z}_{n-1}-\log \beta\right)\right)+(1-\beta) \iint_{y>x-\log \beta}(x-\log \beta) x f(x) f(y) d x d y+ \\
& (1-\beta) \iint_{y>x-\log \beta} y x f(x) f(y) d x d y
\end{aligned}
$$

where $\mathrm{f}$ denotes a standard logistic density

(i.e $f(x)=\frac{e^{-x}}{\left(1+e^{-x}\right)^{2}}$ ) After some manipulation

gives

$$
E\left(\mathbf{Z}_{n} \mathbf{Z}_{n+1}\right)=\frac{\beta \pi^{2}}{3}+(1-\beta) \varphi(\beta)
$$

Where

$$
\varphi(\beta)=\int_{0}^{\infty} \frac{\log \omega \log (1+\beta \omega)}{(1+\omega)^{2}} d \omega
$$

The integral (2.9) may be evaluated numerically for various values of $\beta$. The correlation between $\mathbf{Z}_{n}$ and $\mathbf{Z}_{n+1}$ is then given by

$\rho\left(\mathbf{Z}_{n} ; \mathbf{Z}_{n+1}\right)=4 \frac{E\left(\mathbf{Z}_{n} \mathbf{Z}_{n+1}\right)}{\alpha^{j} \pi^{2}}$

A simple computation gives

$$
\begin{aligned}
& P\left(X_{n+1}>x_{n}\right)=P\left(\mathbf{Z}_{n+1}>z_{n}\right) \\
& =\frac{(1+\beta)}{2}
\end{aligned}
$$

Further, that

$$
\begin{aligned}
& P\left(X_{n+2}>x_{n}\right)=P\left(\mathbf{Z}_{n+2}>z_{n}\right)=\frac{\left(1+\beta^{2}\right)}{2} \\
& P\left(X_{n+3}>x_{n}\right)=P\left(\mathbf{Z}_{n+3}>z_{n}\right)=\frac{\left(1+\beta^{3}\right)}{2}
\end{aligned}
$$

Like (2.10), these may be proved by expressing $\mathbf{Z}_{n+1}, \mathbf{Z}_{n+2}$ in terms of $\mathbf{Z}_{n}$. An unusual feature of the calculation is that the probabilities, conditional on taking a particular branch, are sometimes much more complex than the final answers above. Results (2.10)-(2.12) yield convenient consistent estimators of $\beta$ [2] Thus, for example, writing $U_{n}=1$ for $X_{n+1}>X_{n}$, and 0 otherwise, we find that a consistent estimator of $\beta$ is $\left(\frac{4}{n} \sum_{i=0}^{n} U_{i}\right)-1$. The related observation that $P\left(\left(\mathbf{Z}_{n} \mathbf{Z}_{n+1}>0\right)=\frac{(1+\beta)}{2}\right.$

does not seem to be as useful for estimation purposes (since $\alpha$ and $\sigma$ will be unknown). Based on a particular 
realization $\left\{X_{0}, X_{1}, X_{2}, \ldots . . X_{n}\right\}$ of the basic logistic process [9], convenient consistent estimators are available for all three parameters. Natural candidates are

$\widehat{\mu}=\frac{1}{n+1} \sum_{i=0}^{n} X_{i}$

$\widehat{\alpha}=\left(\frac{3}{\pi^{2}(n+1)} \sum_{i=0}^{n}\left(X_{i}-\widehat{\mu}\right)^{2}\right)^{\frac{1}{2}}$

$\widehat{\beta}=\left(\frac{2}{n} \sum_{i=0}^{n} U_{i}\right)-1$

where $U_{i}$ is defined in the previous paragraph. Alternative estimators for $\alpha$ and $\sigma$ can be obtained using order statistics. Since the median of $\mathrm{F}(0, \sigma)$ is $\sigma$ and the interquartile range is $\alpha \log 16$, consistent estimators can be found from the sample median and inter quartile range. It is considered that the sampling distributions of all these estimators in [4]. Inspection of (2.6) leads to the realization that the joint distribution of $\left(X_{n-1}, X_{n}\right)$ will be singular. In fact there is positive probability that the sequence $\left(X_{n}-X_{n+1}\right)$ contains ties. A straight forward modification in (2.4) will eliminate this potentially troublesome phenomenon. Instead of using the same value of $\beta$ at every stage, a random of $\beta$ is generated. The resulting logistic process is discussed in section 4

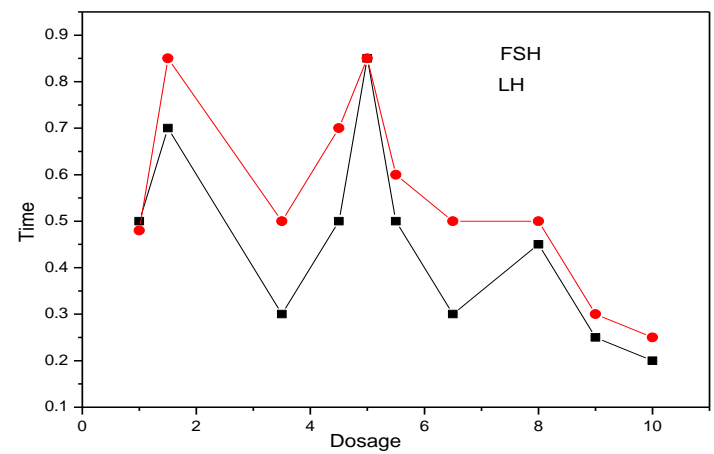

Figure.1 Concentration of LH(Red) and FSH(Black) on lamprey GnRH-III
As an application of these logistic process models we have fitted a set of data. The analysis of the data on the lamprey GnRH - III in LHIFSH concentrations were transformed logarithmically, and differenced. Since the LH has shown a fairly steady downward trend against the FSH. (in figure 2.1) These transformations will fit in a power-logistic process [9].

\section{CONCLUSION}

A mathematical model, based on clinical data has been constructed with a statistical system approach, with the aim of analyzing the performance, and predicting the effects that possible readjustments and/or interventions on the structure may produce on it .When amphibians were infused with doses of GnRH-III , gonadotropin secretion was increased in a dosedependent manner with a greater increase in LH than FSH concentrations.The contradictory results obtained by different laboratories, may be explained by experimental condition, the influence of the presence or absence of steroid in the in vivo models, and data interpretation . Undoubtedly, more research is needed to clarify the existence of GnRH-III or a FSHRF that may be involved in the differential secretion of gonadotropins in mammals.

\section{REFERENCES}

[1] Amstalden, M., D. A. Zieba, M. R. Garcia, R. L. Stanko, T. H. Welsh, Jr., W. H. Hansel, andG. L. Williams. 2004. Evidence that lamprey GnRH-III does not release FSH selectively in cattle. Reproduction 127: 25-42.

[2] Arnold,B. C. and Robertson ,C. A . (1989) Autoregressive logistic processes. J . Appl. Prob. 26 524-521.

[3] Johnson, N. L. And Kotz ,S . (1970) Distributions in Statistics : Continuous Univariate Distribution, Vol. II. Wiley, New York

[4] Lakshmi S., Stochastic Model for Stress Effects of Homeostatic Systems, The Journal of the Indian Academy of Mathematics, 26(2), 2004

[5] Lewls, P. A. W. (1985) Some simple models for continuous variate time series. Water Resources Bull. 21 , 625-644.

[6] McCann, S. M., and W. H. Yu. 2001. FSH-releasing peptides. US Patent 6,200,471.

[7] Padmanabhan, V., F. J. Karsch, and J. S. Lee. 2002 Hypothalamic, pituitary and gonadal regulation of FSH. Reproduction (Cambridge, England) Supplement 59: 6782.

[8] Rao,P . S. AND Johnson D. H. (1988) A first-order A R model for non-Gaussian time series. Proc. IEEE Int. Conf. on ASSP. 2, 1524-1527.

[9] Yeh H. C., Arnold, B. C. AND Robertson, C. A. (1988) Pareto processes. J. Appl. Prob. 25, 291-201. 521

[10] Yu, W. H., S. Karanth, C. A. Mastronardi, S. Sealfon, C. Dean, W. L. Dees, and S. M.McCann. 2002. Lamprey GnRH-III acts on its putative receptor via nitric oxide torelease follicle-stimulating hormone specifically. Experimental Biology and Medicine Maywood, N.J 227 786-792. 


\section{APPENDIX}

A parameter family of distribution which may play the role of

$\mathrm{G}$, the distribution of $\beta$ in section 2 , is provided by the power function distributions. Specifically

$$
G_{\alpha}(\beta)=\beta^{\alpha}, 0<\beta<1
$$

where $\alpha>0$. If this distribution is substituted in (2.2) and (2.5) the following expression for the joint survival function of $\left(\mathbf{Z}_{n} \mathbf{Z}_{n+1}\right)$ in the standard process is obtained:

$$
P\left(\mathbf{Z}_{n}>z_{n}, \mathbf{Z}_{n+1}>z_{n+1}\right)=\frac{\left(1+\beta \exp \left(z_{n+1}\right)\right)}{\left(1+\exp \left(z_{n+1}\right)\right)\left(1+\left[\exp \left(z_{n}\right)\right]\right)}, z_{n}>z_{n+1}
$$$$
5.2
$$

And for $z_{n} \leq z_{n+1}$

$$
\left(1+\exp \left(z_{n+1}\right)\right)^{-1}-\frac{1}{\alpha+1} \frac{\left(\exp (\alpha+1) z_{n} \exp \left(-\alpha z_{n}\right)\right)}{\left(1+\exp \left(z_{n+1}\right)\right)\left(1+\left[\exp \left(z_{n}\right)\right]\right)}
$$

It is evident that the joint distribution of $\left(\mathbf{Z}_{n} \mathbf{Z}_{n+1}\right)$ is absolutely continuous. For reference the corresponding rather cumbersome expression for the joint density function is included.

$$
f_{z_{n} z_{n+1}}\left(z_{n}, z_{n+1}\right)=\frac{\left(\exp -\left(z_{n}+z_{n+1}\right)\right)}{(\alpha+1)\left(1+\exp \left(z_{n+1}\right)\right)^{2}\left(1+\left[\exp \left(z_{n}\right)\right]\right)^{2}}, z_{n} \leq z_{n+1}
$$

While for $z_{n} \leq z_{n+1}$

$$
f_{z_{n, p+1},}\left(z_{n}, z_{n+1}\right)=\frac{\exp \left(-(1-\alpha) z_{n}+(1+\alpha) z_{n+1}\right)\left(1+\frac{\alpha}{\alpha-1} \exp \left(z_{n}\right)\right)\left(1+\frac{\alpha}{\alpha-1} \exp \left(z_{n+1}\right)\right)}{(\alpha+1)\left(1+\exp \left(z_{n+1}\right)\right)^{2}\left(1+\left[\exp \left(z_{n}\right)\right]\right)^{2}}
$$

Note that for the power-logistic process the mean of the distribution $\mathrm{G}$, denoted by

$\mathrm{b} \in(0,0.9)$ is a simple function of $\alpha$ :

$$
\begin{aligned}
& b=\frac{\alpha}{\alpha-1} \\
& \hat{\alpha}=\frac{\left(\frac{4}{n} \sum_{i=0}^{n} U_{i}\right)-1}{4-\left(\frac{4}{n} \sum_{i=0}^{n} U_{i}\right)}
\end{aligned}
$$

Where $U_{n}$ is defined in (2.11). 